Cahiers « Mondes anciens »

ANCIENS

Histoire et anthropologie des mondes anciens

5 | 2014

Maudire et mal dire : paroles menaçantes en Grèce ancienne

\title{
Efficacité et temporalité de l'invective et de la satire dans la poésie grecque
}

Ralph M. Rosen

\section{CpenEdition}

Journals

Édition électronique

URL : http://journals.openedition.org/mondesanciens/1235

DOI : $10.4000 /$ mondesanciens. 1235

ISSN : 2107-0199

Éditeur

UMR 8210 Anthropologie et Histoire des Mondes Antiques

Référence électronique

Ralph M. Rosen, «Efficacité et temporalité de l'invective et de la satire dans la poésie grecque »,

Cahiers « Mondes anciens » [En ligne], 5 | 2014, mis en ligne le 14 février 2014, consulté le 20 avril 2019.

URL : http://journals.openedition.org/mondesanciens/1235; DOI : 10.4000/mondesanciens. 1235

Ce document a été généré automatiquement le 20 avril 2019

\section{c) (i) $९$}

Les Cahiers «Mondes Anciens » sont mis à disposition selon les termes de la licence Creative

Commons Attribution - Pas d'Utilisation Commerciale - Pas de Modification 4.0 International. 


\title{
Efficacité et temporalité de l'invective et de la satire dans la poésie grecque
}

\author{
Ralph M. Rosen
}

1 Parmi tous les genres littéraires traditionnels, ceux reposant sur l'invective, la raillerie et la satire pourraient bien s'avérer être les plus problématiques ${ }^{1}$. Ainsi, les véhémentes satires $^{2} \mathrm{du}$ célèbre poète grec Archiloque agacèrent déjà à l'époque nombre de ses contemporains $^{3}$, et froissèrent plus tard également jusqu'à Platon et Aristote qui exprimèrent leur malaise face à ces propos virulents et cette parole transgressive pourtant si caractéristiques de ce registre littéraire ${ }^{4}$. Tout ce qui contribue au succès de cette poésie de l'insulte et de la dérision - sa rhétorique belliqueuse, par exemple, ainsi que son caractère prétendument vertueux, son indignation délibérément affichée, sa prédilection pour toute transgression linguistique et parfois même morale - fait ainsi obstacle à la compréhension et à l'analyse de ces textes. Les différents publics de ces œuvres sont en effet happés dans ce flot d'invectives à la source de ce rire qui dérange, tandis que l'auteur de ces œuvres a pourtant toujours en vue une cible bien précise qu'il entend bien corriger voire abattre. Or, une personne se sentant ainsi visée par cette parole injurieuse, peut elle-même s'offusquer de tant de véhémence et passer alors à l'offensive. D'autres, par contre, demeureront insensibles aux vitupérations et à la démarche démagogique du poète, ou ne percevront pas, tout simplement - et là réside alors le danger - l'ironie d'un tel discours pourtant à la base de l'humour satirique. Enfin, cette poésie de l'invective demeure si ancrée dans le temps et dans l'espace qu'elle ne s'exporte pas facilement vers d'autres publics ni vers d'autres lecteurs situés ailleurs, dans d'autres lieux et à d'autres époques. Les personnes visées et concernées par ces œuvres ont en effet une durée de vie limitée. Les mots cependant perdurent, et des lecteurs temporellement éloignés de ces œuvres peuvent ainsi continuer à se confronter aux railleries et aux injures de l'auteur disparu, lui, depuis des siècles. La réception et la signification de ce type de poésie changent alors radicalement et conformément à l'évolution des rapports qu'entretiennent la cible visée par ces œuvres et le public. 
2 Les lecteurs de l'œuvre d'Aristophane se retrouvent ainsi fréquemment confrontés à cette situation. En effet, les très nombreux personnages dont se moque Aristophane dans ses pièces demeurent de véritables énigmes pour quiconque est situé hors du contexte historique de l'époque qui les a vus naître. Les lecteurs successifs de cette œuvre doivent donc bien souvent se démener pour acquérir toutes les connaissances nécessaires à la compréhension, ne serait-ce que rudimentaire, de la verve satirique de l'auteur envers ces individus si connotés historiquement. Par conséquent, comment apprécier la dérision dès lors qu'il est impossible de saisir pleinement l'importance et le rôle des individus raillés au sein de pièces qui reflètent l'urgence du moment? Ou pour le dire encore autrement : comment interpréter ces textes si nous présumons uniquement que la satire de l'auteur n'est vraiment pertinente et efficace que lorsqu'un public la comprend comme étant en effet dirigée contre des personnes et des problèmes réels, situés eux-mêmes dans le « ici et maintenant » de l'auteur et de son public ?

3 Se poser cette question revient donc à s'interroger sur la façon dont nous devons procéder pour trouver un sens à ces œuvres satiriques et comment, surtout, nous pouvons parvenir à réconcilier le caractère à la fois circonstanciel et intemporel de ces textes. Qu'advient-il en effet du caractère tendancieux de ces œuvres (c'est-à-dire si l'on admet que ces dernières agissent en effet directement contre des personnes réelles dans un monde tout aussi réel, et poursuivent donc bien aussi un objectif « sérieux ») alors que la satire perd en effet de son efficacité originelle au fil du temps?

4 Telles sont donc les questions auxquelles je m'intéresserai tout au long de cet article en m'appuyant plus particulièrement sur les œuvres des premiers poètes satiriques, Archiloque et Hipponax, ainsi que sur les pièces d'Aristophane dont le penchant pour la dérision et l'insulte doit beaucoup à la poésie en vers iambiques de ses prédécesseurs.

Comme nous le verrons également, ces questions qui semblent de prime abord ne concerner que les œuvres de quelques poètes ayant vécu à une époque bien précise, concernent cependant toute œuvre littéraire de registre satirique (y compris celles de notre époque), et mon propos sera alors tout aussi bien d'ordre littéraire que d'ordre théorique. De plus, j'aimerais avancer que c'est bien en effet cette question de l'efficacité et de la pertinence de la satire et de l'invective que soulèvent tous les textes appartenant à ce registre : quel est en effet le but primordial d'un discours ad personam si belliqueux et si fondamentalement irrévérencieux?

6 Commençons avec la querelle entre Archiloque et Lycambe au sujet de ses filles, une histoire célèbre dans l'Antiquité et emblématique de la verve satirique d'Archiloque ${ }^{5}$.

7 La fille de Lycambe, Néoboulè, devait donc épouser Archiloque mais pour d'obscures raisons, Lycambe décida d'annuler ce mariage. Furieux, Archiloque écrivit alors des poèmes insultants envers la famille de Lycambe tout entière, accusant entre autres ses filles de se livrer à des pratiques sexuelles défendues dans des lieux sacrés. La plupart des informations que nous détenons sur cette querelle proviennent davantage de témoignages que des quelques fragments de son œuvre qui nous sont parvenus, mais la publication de l'épode de Cologne nous a depuis offert une meilleure vue d'ensemble sur cette poésie de l'invective ${ }^{6}$. Dans cet extrait, l'orateur, et donc probablement le poète luimême, se retourne de façon très agressive contre Néoboulè pour tenter à présent de séduire sa sœur :

...Neoßoú $\lambda \eta[v$

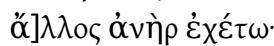

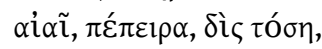




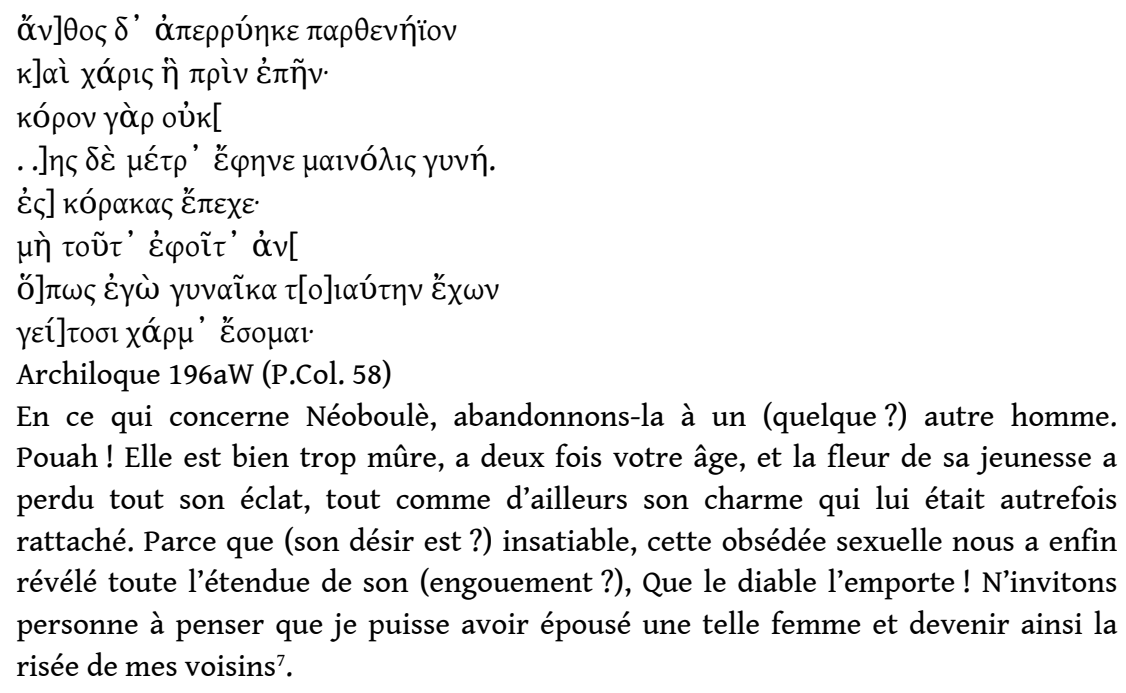

8 Au siècle suivant, un autre poète prétendument en colère, Hipponax, choisit également d'insulter certains individus de son époque. Boupalos, cible préférée d'Hipponax, est ainsi aussi célèbre que Lycambe chez Archiloque, tous deux étant alors devenus des modèles classiques de têtes de Turc dans ce type de poésie. La querelle d'Hipponax avec Boupalos concerne également une femme, Arète, qui sembla préférer Boupalos au poète. Aucun des fragments ne nous offre beaucoup de détails sur cette petite histoire, mais le fr. $12 \mathrm{~W}$ (= $20 \mathrm{Dg}$ ) sous-entend cependant très clairement que tous deux étaient impliqués dans quelque échange très salace ${ }^{8}$ :

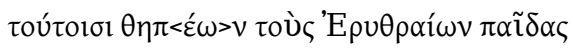

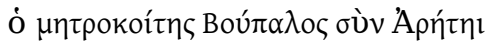

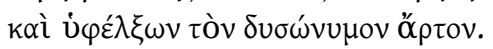

Et ce connard de Boupalos avec Arète, trompant les Erythréens avec ces mots (par ces moyens ?) et s'apprêtant à retrousser son maudit prépuce...

Dans le fr. $19 \mathrm{~W}$ (= $33 \mathrm{Dg}$ ), la personne visée ici n'est pas identifiée, mais ces propos pourraient fort bien s'adresser à Boupalos :

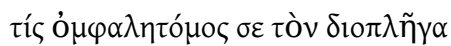

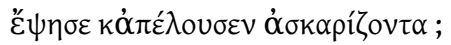

Quel coupe-nombril t'a donc essuyé et lavé pendant que tu te tortillais de douleur, espèce de cinglé ?

Enfin, ce dernier passage illustre bien également le caractère injurieux de la verve d'Hipponax qui prend ici pour cible le peintre Mimnès, par ailleurs inconnu (fr. $28 \mathrm{~W}=$ $39 \mathrm{Dg})$ :

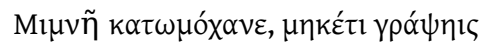

ö

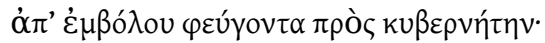

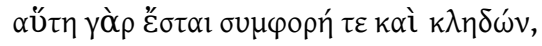

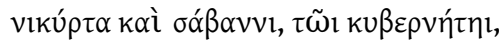

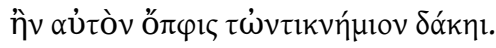

Mimnès, toi qui bâilles à t'en décrocher la mâchoire,

ne peins plus sur le côté à plusieurs bancs d'un trirème

un serpent qui va de la rame

au timonier ; car c'est là un mauvais présage pour le

timonier, toi esclave né d'une esclave,

et... si le serpent le mord au tibia. 
11 Comment le public est-il censé répondre à ce genre de poésie ? D’autre part, de quel public devons-nous tenir compte - de celui du poète, ou de ses différents publics qui viendront bien après lui ? C'est bien là, et comme je l'ai souligné plus haut, que réside toute la difficulté. Parce que la dérision et les insultes au sein de ce genre de poésie concernent effectivement des individus contemporains de l'auteur et des différents publics de son œuvre, ces questions relevant de l'efficacité de la satire sur les publics postérieurs prennent évidemment ici toute leur importance. Même l'approche la plus naïve qui consisterait à prendre le poète au mot - et donc aussi à présumer que son œuvre met véritablement en scène ses réelles inimités et son désir sincère de leur nuire - ne semble significative que lors du vivant du poète. Le public contemporain qui connaît les personnes visées par le poète dans ses vers, ou qui a du moins entendu parler d'elles, peut en effet participer pleinement à ce rire vengeur si caractéristique du succès de cette poésie de la dérision, et ce genre de raillerie semble alors bien "agir » en temps réel. Cette efficacité de la moquerie sur le public de l'époque - qui me semble être davantage un prétexte plutôt qu'une réalité nécessaire ${ }^{9}$ - peut alors s'expliquer de différentes façons. Par exemple, les spectateurs de ces pièces riront volontiers et se feront donc complices du poète s'ils croient en effet que l'œuvre de ce dernier représente bien la réalité d'un « ici et maintenant » qu'ils partagent avec le poète. Ainsi, pourraient-ils fort bien s'écrier à l'unisson, et de tout cœur avec le poète: "Oui, Hipponax, Boupalos ne mérite pas Arete! Oui, Archiloque, il est tout à fait scandaleux que Lycambe ait annulé ton mariage avec Néoboulè ! Nous te soutenons et nous comprenons ton indignation, et tous ces gens que tu vilipendes ici devraient en effet avoir honte!». Naissant donc d'une intention bien spécifique, ces invectives deviennent en outre ici « actes de parole », et dès qu'un public perspicace répond par le rire, nous pouvons affirmer que le poète est arrivé à ses fins.

12 Mais sur quoi porte le rire quand le poète et les individus qu'il a pris pour cibles ont depuis longtemps disparu? Pourquoi un auditeur ou un lecteur se soucie-t-il autant des personnages de ces poèmes et trouve-t-il alors ce genre de moquerie si divertissant?

13 Ce genre de problème ne semble pas affecter autant les textes non satiriques. Par exemple, CEdipe, Énée et Hamlet, peuvent somme toute, être aisément « interprétés » par un public d'une autre époque. Mais l'œuvre satirique, elle, résiste fermement à la diversité des interprétations puisqu'en effet, cette dernière est parcourue, voire menée, par une seule et unique voix, solipsiste de surcroît, revendiquant des intérêts personnels et le désir d'exprimer une "vérité » intimement liée à son époque. Cette voix auctoriale ne saurait alors accueillir n'importe quelle "interprétation », venant de quiconque situé dans un autre temps.

Cette rhétorique de l'indignation et de l'urgence temporelle si caractéristique de la satire repose donc en grande partie sur le principe de synchronie. L'œuvre satirique ne tolère en effet aucun intermédiaire. Ses préoccupations sont franches et directes et l'œuvre n'expose que ce qu'elle souhaite révéler. Pourtant, les auteurs satiriques sont également des acteurs. Leur agression est en effet mimétique et artificielle, et ce aussi bien pour plaire au public que pour censurer les personnes qu'ils ont prises pour cibles. Bien que leur poésie revendique immédiateté et franchise, leur désir de vouloir agir de façon préméditée sur la réponse d'un public bien précis, exige en effet de ces poètes qu'ils respectent eux-mêmes certaines traditions littéraires concernant le discours performatif. Or ces traditions possèdent elles-mêmes leur propre histoire très complexe, et ces auteurs se doivent ainsi de respecter également cette diachronie qui se trouve alors elle-même 
aussi profondément inscrite au sein de leurs œuvres, que ce soit au niveau linguistique, au niveau thématique ou encore au niveau intertextuel. Ce respect inévitable de la diachronie au sein même d'une poésie ne reposant de prime abord que sur le principe de synchronie, crée donc une tension qui peut s'avérer choquante, voire paradoxale.

Les revendications répétées du satiriste quant aux motivations de son œuvre ne laissent en effet que peu de place à une éventuelle réflexion diachronique. En s'attardant sur des considérations purement littéraires et d'histoire générique, toute réflexion diachronique pourrait en effet menacer la subjectivité du satiriste si bien construite au sein de son œuvre. La satire se veut ainsi être en temps réel, un engagement franc et direct, c'est-àdire sans aucun intermédiaire, entre l'auteur et son public, au sujet de questions ayant forcément une incidence sur la vie de l'auteur telle qu'il a lui-même choisi de la représenter dans son œuvre. Dans ce contexte, tenter de déceler une quelconque "vérité" satirique dans ces propos ne reviendrait qu'à énoncer des platitudes comportement lui-même alors considéré comme "mauvais", et donc tout simplement présenté aussi comme tel au public. Par conséquent, la logique de la satire va à l'encontre de tout concept d'« intemporalité » et d'« universalité »: la satire n'évolue a priori que dans le moment présent, elle n'entretient aucun lien avec le passé et l'avenir, et n'a aucune prétention littéraire qui pourrait venir faire obstacle à la ridiculisation recherchée ${ }^{10}$.

Cependant, la réalité est bien différente. Les poètes satiriques usent en effet de multiples astuces non seulement pour embellir leurs attaques d'artifices verbaux très stylisés, mais aussi pour situer leur œuvre dans une tradition littéraire qui est également diachroniquement construite.

17 Ce heurt entre synchronie et diachronie soulève donc de vastes questions aussi bien théoriques que pratiques avec lesquelles nous sommes toujours aux prises aujourd'hui en tant qu'auditeurs et disciples de ce genre de poésie.

18 Que reste-t-il en effet au public de ses œuvres si, par exemple, une conscience diachronique tourne elle-même en raillerie la contemporanéité même de la satire, menaçant ainsi, et via le comique, de compromettre voire de saper, la valeur de vérité de ses propres allégations? Le public, et nous-mêmes, pouvons-nous alors jamais énoncer clairement le «sens » de tout propos satirique quand les auteurs eux-mêmes ont depuis longtemps compris que le comique naît justement de cette déstabilisation du sens et non de sa clarification ${ }^{11}$ ?

Or c'est bien cette question que Plutarque semble également avoir à l'esprit dans ce passage des Propos de table (Symposiakôn), consacré aux «divertissements auxquels recourir pendant les banquets », où Diogénianos soulève le problème de l'interprétation des plaisanteries et de la bouffonnerie dans la comédie ancienne :

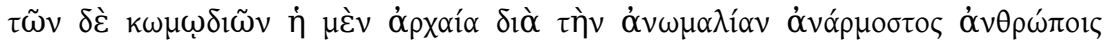

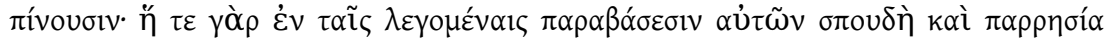

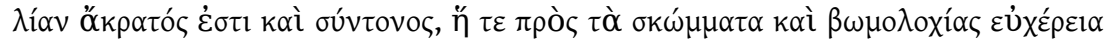

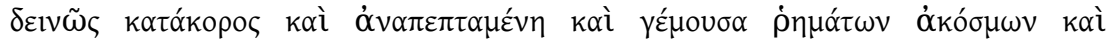

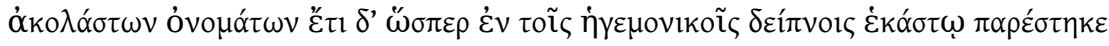

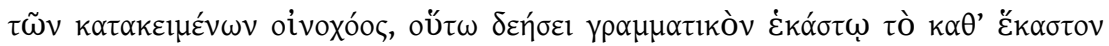

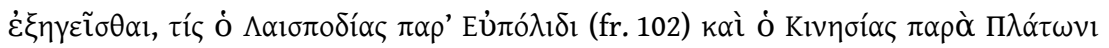

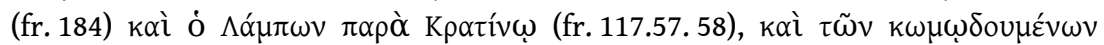

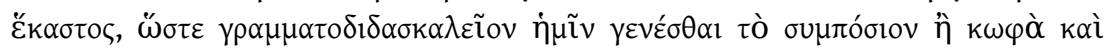

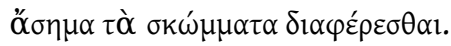


De tous les types de comédies, l'Ancienne, qui manque souvent de cohérence

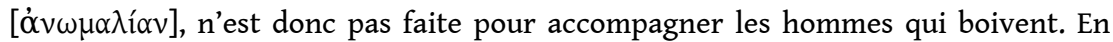

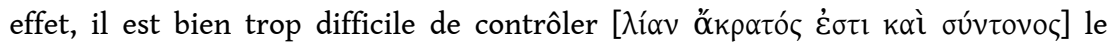

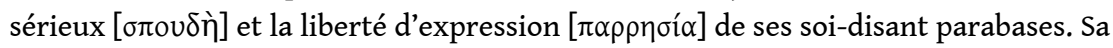
complaisance pour les plaisanteries et les bouffonneries est terriblement extravagante et impudente, et de ses propos fusent souvent d'indécentes et d'effrénées expressions. Comme lors des banquets des gens riches et célèbres où derrière chaque invité se tient un échanson mis à sa disposition, nous-mêmes

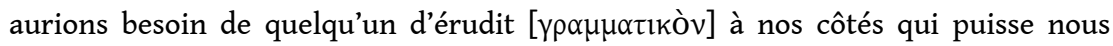
expliquer chaque référence. Par exemple, qui était Laispodias dans l'œuvre d'Eupolis [F 107], Cinésias chez Platon [F 200], Lampon chez Cratinos [F 62, 125]

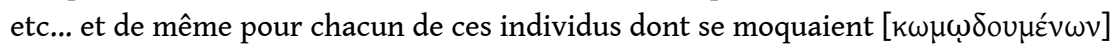
ces auteurs. Notre petite fête ressemblerait alors à une salle de classe où l'on assènerait des plaisanteries $[\sigma \kappa \omega ́ \mu \mu \alpha \tau \alpha]$ sur des oreilles sourdes et ignorantes ${ }^{12}$.

Quiconque se penche aujourd'hui sur un $\sigma \kappa \omega ́ \mu \mu \alpha \tau \alpha$ d'un poète iambique ou sur une pièce d'Aristophane, comprend très bien ici les propos de Diogénianos. D'un certain point de vue - et de celui du poète surtout qui, lui, prétend à une véritable efficacité de ses propos - les $\kappa \omega \mu \omega \delta$ oú $\mu \varepsilon v o l$ ont en effet, pourrions-nous dire, une date de péremption, et sont donc « à consommer » de préférence immédiatement. Cependant, ces œuvres perdurent aussi en tant que véritables textes littéraires et par conséquent, tirent également leur signification de ce rôle, un rôle où les questions de diachronie en viennent donc à éclipser progressivement, voire à effacer totalement, celles liées à la synchronie.

21 Ce processus commence dès que le poète confie aux mots ses insultes et sa raillerie. En effet, le poème est lui-même le premier filtre à travers lequel le public perçoit la dimension synchronique de l'œuvre, et c'est également le poème, comme nous l'avons dit plus haut, qui exerce aussi cette sorte de traction diachronique. Car c'est bien lui qui parvient à attirer l'attention du public sur une certaine esthétique de la raillerie et qui lui dévoile alors aussi, cette histoire poétique et générique à laquelle appartient également le poète, et qui existe donc indépendamment de la temporalité explicite de l'œuvre.

Afin d'illustrer mes propos qui pourraient sembler jusqu'ici un peu abstraits, j'ai choisi un passage de Lysistrata d'Aristophane qui démontre bien comment un personnage issu d'un iambe archaïque et pris pour cible dans son temps et son histoire (et donc pris aussi dans la synchronie de l'œuvre) peut se métamorphoser en un emblème diachronique du registre satirique :

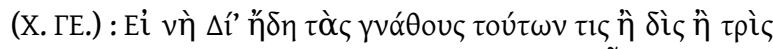

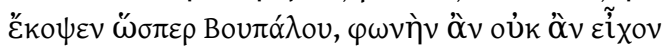

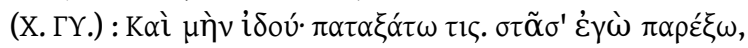

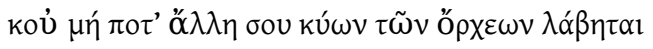

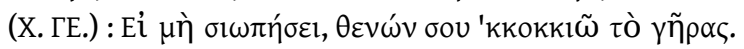

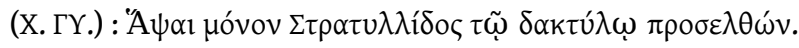

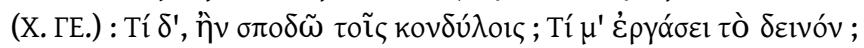

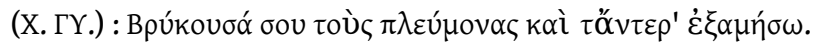

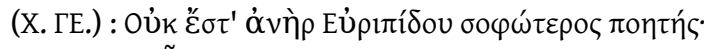

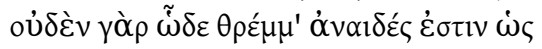

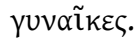

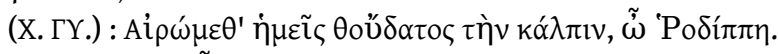

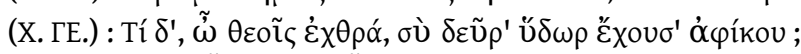

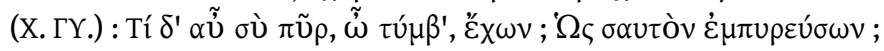

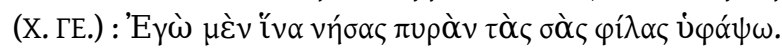

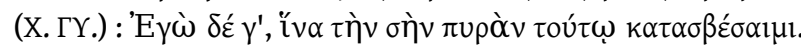

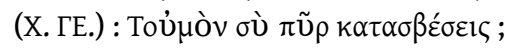




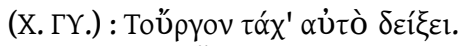

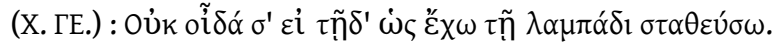

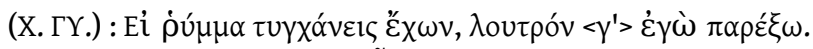

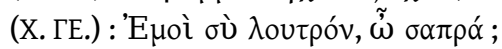

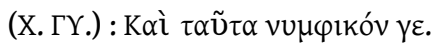

(Lysistrata, 360-380)

Le Coryphée - Si, par Zeus, on leur avait déjà donné deux ou trois coups sur la mâchoire, comme à Boupalos, elles ne pourraient parler.

La Coryphée - Eh bien, me voilà, qu'on me frappe. Je m'y prêterai sans broncher ; mais jamais une autre chienne te happera les bourses.

Le Coryphée - Si tu ne te tais, à coups de bâton, je te ferai partir la peau.

La Coryphée - Touche seulement du doigt Stratyllis, ose approcher.

Le Coryphée - Et si je te mets en poussière avec mes poings? Que me feras-tu de si

terrible?

La Coryphée - En mordant, je t'arracherai poumons et entrailles.

Le Coryphée - Non, il n'est pas de poète plus sage qu'Euripide ; car il n'est pas d'engeance aussi impudente que les femmes.

La Coryphée - Soulevons, nous, nos pots d'eau, Rhodippe.

Le Coryphée - Eh quoi, ennemie des dieux, qu'es-tu venue faire ici avec de l'eau ?

La Coryphée - Et toi donc, avec du feu, vieux tombeau ? Est-ce pour t'incendier toi-

même?

Le Coryphée - Moi, c'est pour entasser un bûcher et mettre le feu sous tes amies.

La Coryphée - Et moi, c'est pour pouvoir avec cette eau éteindre ton bûcher.

Le Coryphée - Tu éteindras mon feu ?

La Coryphée - Le fait va te le prouver.

Le Coryphée - Je me demande si de ce pas, je ne vais pas te griller avec cette torche.

La Coryphée - Si tu as à lessiver par hasard, je te fournirai, moi, un bain.

Le Coryphée - Un bain, à moi, pourriture?

La Coryphée - Un bain nuptial encore.

Cette scène présente un affrontement très conventionnel entre deux chœurs, l'un féminin et l'autre masculin, et ressemble à de nombreuses autres scènes d'invectives chez Aristophane ${ }^{13}$ durant lesquelles la passion théâtrale s'épanche, les caractères coléreux éclatent, les voix hurlent et les accusations fusent. Aucune place n'est faite cependant à la discussion voire à la réflexion sur ce qui est dit dans cette scène et les complaintes de chacun ne semblent guère substantielles. Ces scènes se déroulent généralement à une allure soutenue et les réparties des personnages ne s'adressent qu'aux seuls personnages présents sur scène, et donc uniquement à ceux dont il est précisément question dans la scène qui se joue à ce moment-là sous les yeux du spectateur. Tous les éléments que l'on associe d'ordinaire à la satire sont également présents : indignation qui semble justifiée (avec d'une part, deux antagonistes tout aussi indignés l'un que l'autre, mais selon la mise en scène d'un auteur qui sait d'autre part, orienter nos amitiés dans la « bonne » direction pour que nous puissions, et sans hésitation, prendre parti) ${ }^{14}$, discours menaçant et langage obscène. Le discours des différents personnages doit aussi être enflammé et sembler jaillir directement du cœur du locuteur.

Cependant, et comme souvent avec la satire littéraire, rien n'est pourtant aussi éloigné de la vérité. Les vers sont en effet habilement façonnés par le poète: la métrique est régulière et répétitive, les rimes parfaitement étudiées et la syntaxe hautement élaborée. En bref, deux «réels" ennemis se rencontrant dans l'agora, devant l'étal d'un poissonnier, ne sauraient se quereller de la sorte! Cette forme très étudiée de la parabase peut fournir au public un moment de détente et une raison de rire, et comme telle, elle 
nous fait alors aussi comprendre que ce genre de scène est bien la mimèsis d'une réelle confrontation, et non pas un exemple de cette dernière. Mais dans ce cas, qu'advient-il alors du prétexte de synchronie - ce prétexte selon lequel tout ce qui se passe à ce moment-là sur scène est, dirions-nous, parfaitement "synchronisé » avec la réalité temporelle du public?

De plus, les discontinuités temporelles présentes dans ce genre de scènes proviennent non seulement de la disjonction entre la parole et le langage poétique, mais également de l'origine littéraire et de l'intertextualité très prononcée que tout cela implique.

Je me suis déjà penché dans un autre ouvrage sur ce genre de scènes très stylisées et montré combien cette forme d'invective aristophanesque était redevable aux poètes iambiques $^{15}$ - et surtout à Hipponax - au niveau de la diction et du style, qu'il s'agisse des rimes, des homoioteleuta, des obscénités, de la violence (qu'elle soit menaçante ou jouée) ou des cris, le tout étant mis au service des injures et de la dérision ${ }^{16}$.

Or cette longue tradition littéraire et poétique vient insinuer de la diachronie dans la synchronie et alors même que les revendications impérieuses des personnages semblent, elles, suggérer qu'elles ne souffrent d'aucune intervention et que le public les recevra alors comme telles.

Bien entendu, quelques spectateurs demeureront perplexes devant cette scène se jouant sous leurs yeux ${ }^{17}$. Cependant aucun doute n'est possible sur la maîtrise dont fait preuve Aristophane quant aux stratégies littéraires qu'il emploie ici.

$\mathrm{Au}$ début de ce même passage, le personnage menant le chœur des hommes apostrophe ainsi le public (vers 360-361) : «Si, par Zeus, on leur avait déjà donné deux ou trois coups sur la mâchoire, comme à Boupalos, elles ne pourraient parler ». Ce à quoi répond le personnage féminin du chœur antagoniste : « Eh bien, me voilà, qu'on me frappe. Je m'y prêterai sans broncher; mais jamais une autre chienne te happera les bourses». Boupalos, bien entendu, était la cible préférée d'Hipponax et comme nous le savons d'après les fragments, le poète aimait lui lancer ses piques les plus acérées et ses insultes les plus grossières ${ }^{18}$. Au risque d'exagérer, rappelons cependant que les vers de cette scène de Lysistrata ont été conçus "à dessein » et qu'encore une fois, il est alors peu probable que le public, emporté par le rythme soutenu et la suite ininterrompue d'actions de cette scène, ait eu le temps de se livrer à des considérations d'ordre littéraire. Cependant, l'allusion à Boupalos ne peut qu'évoquer clairement dans l'esprit des spectateurs cette longue histoire littéraire se jouant également derrière cette scène. Le nom de Boupalos, dans la bouche du coryphée du clan masculin, résonne ici comme une insulte tout à fait banale que n'importe quel individu pourrait lancer à son adversaire, et si nous ne savions pas qui était en réalité Boupalos, nous pourrions tout simplement en conclure que ce dernier n'était qu'un simple individu malgré tout célèbre à cette époque pour quelques-unes de ses escarmouches. Mais bien évidemment, et puisque nous savons fort bien en effet qui était Boupalos à cette époque, nous sommes alors parfaitement en mesure de comprendre les réels enjeux de cette scène, à savoir qu'il serait en effet formidable si les deux clans en présence pouvaient ici rejouer cette célèbre querelle littéraire entre Hipponax et Boupalos avec d'un côté, le chœur masculin dans le rôle du poète satirique tout à fait dans son droit (Hipponax), et de l'autre, le choeur féminin dans le rôle de sa vilaine cible (Boupalos). Aucune équivoque ne serait alors possible sur l'issue d'une scène agencée de la sorte et donc aussi sur celui qui en sortirait vainqueur. 
30 Or, dans cette scène, si le clan des femmes semble en effet accepter de jouer gracieusement le rôle de Boupalos (« Eh bien, me voilà, qu'on me frappe. »), la réponse de ces dernières contient aussi une terrible menace, tout à fait inattendue ici : si les hommes osent les frapper, elles-mêmes se refuseront alors à tout rapport sexuel avec eux. Par conséquent, le doute subsiste quant au vainqueur de cette querelle se jouant ici sous les yeux d'un public hésitant lui-même aussi à prendre parti pour l'un ou l'autre clan. Aristophane semble en effet accorder à son personnage d'Hipponax la maîtrise de son psogos tout en offrant aussi à son Boupalos - le clan des femmes - une occasion de remporter la victoire.

31 Au vers 368 , nous retrouvons également une autre allusion littéraire quand le coryphée cite ainsi Euripide : «Non, il n'est pas de poète plus sage qu'Euripide ; car il n'est pas d'animal aussi impudent que les femmes ". Là encore, une telle allusion n'est guère probable lors d'un échange d'insultes. Cependant, ce vers incarne parfaitement ce conflit des différentes temporalités à l'œuvre au sein de ces registres de l'invective et de la satire. En effet, le sens même de la citation équivaut à une insulte car ce "vous, les femmes, êtes toutes impudentes » etc... pourrait très bien en effet être lancé au visage de ces femmes-là et n'être par conséquent justifié que durant cette scène-là. Or ce vers est lourd en sous-entendus littéraires et oblige donc l'auditeur à effectuer un va-et-vient entre hier et aujourd'hui, et ce, même si ce retour en arrière n'est que de très courte durée. En effet, et quand bien même ces mots d'Euripide auraient été prononcés la veille de la représentation de Lysistrata, leur objectif en aurait été le même: détourner l'attention du spectateur vers un temps autre que celui de cette scène se jouant sous ses yeux. Un tel mouvement diachronique est certes infime mais l'allusion à Euripide situe cependant la scène en question dans un contexte littéraire qui lui est propre, et par conséquent, renforce et garantit par là-même aussi la diachronie même de ce mouvement. En effet, ce genre d'invective provient non seulement de cette poésie en vers iambiques issue d'une longue tradition, mais l'allusion d'Aristophane à d'autres poètes se consacrant à d'autres genres littéraires, vient également démentir ce soi-disant principe de synchronie sur lequel compte pourtant aussi la comédie satirique.

Dans ce passage, et ailleurs dans ses pièces, Aristophane semble donc reconnaître que la satire et la raillerie demeurent problématiques en raison de ce conflit perpétuel entre perspective diachronique et perspective synchronique. Après tout, la satire exprime surtout un désir de nuire et se refuse à toute critique. À tort ou à raison, elle attaque et censure en effet des individus, use d'un langage infâme, profite davantage des conflits que des accords interpersonnels, et exige enfin que son public ne remette aucunement en question la réalité qu'elle lui présente ${ }^{19}$. Il n'est donc pas surprenant qu'un certain public soit lui aussi mal à l'aise vis-à-vis des transgressions que la satire se permet quant aux bienséances sociales et esthétiques.

33 Aristophane s'est également penché sur ce problème dans la parodos des Grenouilles (316-459) situant alors la comédie satirique dans le monde des rites religieux ${ }^{20}$. En insistant sur ce lien avec la religion, Aristophane parvient ainsi à isoler clairement deux types de publics: d'un côté un public naïf et "non-initié » réagissant par le rire à l'aischrologie, aux moqueries obscènes et aux insultes et ne considérant alors ces dernières que comme de simples phénomènes de cet «ici et maintenant » auquel luimême appartient; et de l'autre, un public «initié», et comprenant fort bien que des enjeux bien plus sérieux sont ici à l'œuvre. 

font très bien comprendre que la comédie satirique ne s'adresse qu'à un public d'initiés, et que les autres n'ont rien à faire là, qu'ils n'ont surtout aucun droit de s'indigner de cette parodie comique.

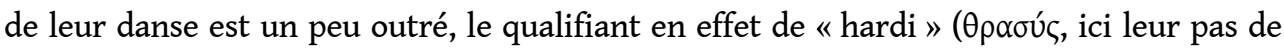

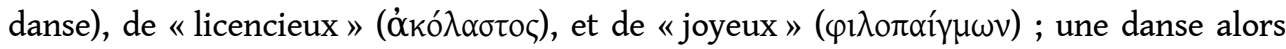

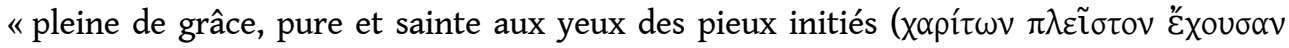

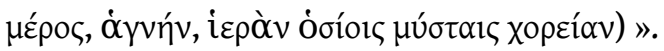

Aux vers 353-370, les Mystes affirment cependant, et de façon très explicite, que la comédie satirique requiert de son public un certain « savoir ", voire la compréhension de l'histoire littéraire et de la poétique de ce genre :

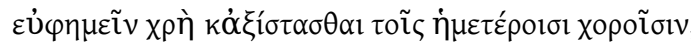

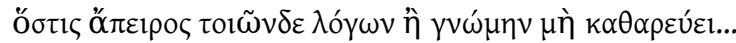

Que l'on se recueille et que cède la place à nos chœurs qui n'est pas versé dans un pareil langage ; qui n'est pas pur d'esprit... (v. 352-354)

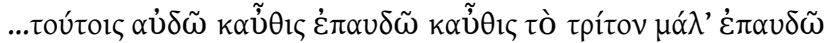

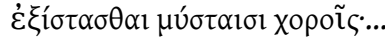

À ceux-là je dis, je redis, je dis bien fort pour la troisième fois de céder la place aux chœurs des mystes. (v. 369-370)

Du vers 354 au vers 369 , le chœur offre également une panorama de tous ceux qui pourraient devenir la cible d'un poète satirique, et semble alors suggérer à ces derniers de ne pas assister à ce genre de spectacles s'ils ne peuvent supporter d'y être ridiculisés. Ce raisonnement est en outre explicité dans le reste de leur chant où les Mystes ne cessent de répéter que la raillerie et les injures, accompagnées par des danses et des jeux, représentent plus ou moins ce dont il est question ici, et donc bien aussi, «ce qu'ils ne cessent de faire » :

$\chi \omega ́ \rho \varepsilon l v v v \pi \tilde{\alpha} \varsigma \dot{\alpha} v \delta \rho \varepsilon i ́ \omega \varsigma$

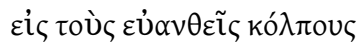

$\lambda \varepsilon\llcorner\mu \omega ́ v \omega v \dot{\varepsilon} \gamma \kappa \rho \circ u ́ \omega v$

$\kappa \dot{\pi} \pi l \sigma \kappa \omega \dot{\pi} \tau \omega \nu$

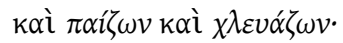

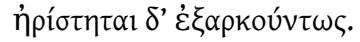

Que chacun donc s'avance résolument dans les replis fleuris des prairies, frappant

$\mathrm{du}$ pied le sol, décochant railleries, plaisanterie, moquerie. Nous avons déjeuné suffisamment. (v. 372-373)

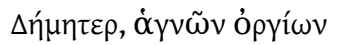

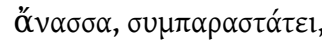

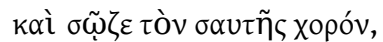

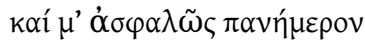

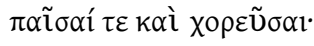

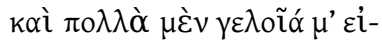

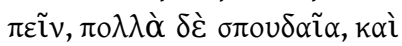

$\tau \tilde{\eta} \varsigma \sigma \tilde{\eta} \varsigma \dot{\varepsilon} \circ \rho \tau \tilde{\eta} \varsigma \dot{\alpha} \xi \hat{i} \omega \varsigma$

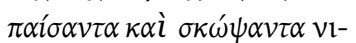

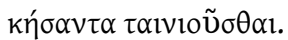

Déméter, reine des saintes, assiste-nous, sauve ton chœur. Fais qu'en sécurité toute la journée, je puisse me livrer aux jeux et aux danses. Que je tienne bien des propos plaisants, bien des propos sérieux ; qu'après avoir badiné et raillé d'une manière digne de ta fête, vainqueur, je porte les bandelettes [de victoire]. (v. 385-393) 
cour illustrer leurs propos (puisqu'un tel passage ne semble pas avoir ic d'autre motivation que celle-là), du vers 416 au vers 430, les Mystes choisissent de prendre en exemple trois de leurs contemporains. Le premier, Archédémos, accusateur durant le procès des généraux de la bataille des Arginuses :

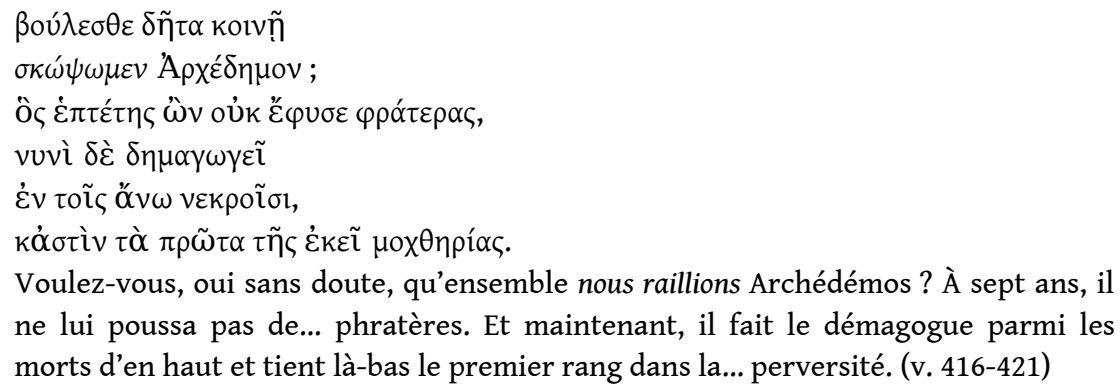

Après Archédémos, les Mystes insultent ensuite ouvertement deux autres de leurs contemporains : Clisthène (son fils n'étant pas non plus épargné par leurs grossièretés) et Callias (vers 422-430), qui se retrouve, lui plus encore, accablé des pires insultes. Dans ce passage, le chœur des Mystes attire surtout l'attention sur ce conflit entre diachronie et synchronie intrinsèque à la satire. Comme je l'ai en effet suggéré, la comédie satirique se moque, en temps réel, de personnes tout aussi réelles. Cependant, les raisons qui soustendent la raillerie, les insultes et les obscénités, de même que les formes mimétiques déployées pour les mettre en scène, sont surdéterminées a priori par plusieurs facteurs génériques et historiques qui contribuent à repousser ce prétexte de pure synchronie. Le raisonnement tacite et dissimulé ici derrière les paroles et les pas de danse du chœur des Mystes pourrait donc se comprendre ainsi : la comédie dramatique (telle que celle dont ils font ici partie) ridiculise les gens uniquement parce que c'est le genre lui-même, et lui seul, qui réclame la raillerie. Certes, de réelles "mauvaises gens " méritant d'être ridiculisées peuvent être une source puissante d'inspiration pour la satire. Cependant, c'est le genre lui-même qui part en quête de ce matériau comique, et non le contraire. Ou pour le dire encore autrement: ce n'est pas l'existence même de ces individus-là qui donne naissance au spectacle comique. Bien avant qu'Archédémos ou que Clisthène n'arrivent en effet sur scène à Athènes, une longue tradition poétique et diachronique de « la raillerie, du mot plaisant et de la satire » existait déjà, et n'attendait donc que cette mise en action synchronique. qui surviennent dans le récit:

On pourrait dire que nous avons, devant nous, deux événements : celui qui nous est raconté dans l'œuvre, et celui de la narration elle-même (et nous participons nousmêmes à ce dernier, comme auditeurs-lecteurs). Ces événements se déroulent à des moments différents (par leur durée, aussi) et en des lieux différents. Simultanément, ils sont inséparablement réunis dans un événement unique, mais compliqué, que nous pourrions désigner comme l'œuvre dans sa plénitude événementielle [...]. Si nous percevons cette plénitude dans son entité indivisible, nous comprenons en même temps tout ce qui différencie les éléments dont elle est constituée ${ }^{21}$.

Cependant, et dans le cas de la satire, comprendre les différents éléments qui constituent sa " plénitude » affecte également le sens même de l'œuvre. Cette diversité se heurte en effet à l'intention immédiate du satiriste et tend à émousser la force de toute revendication (morale ou didactique) que ce dernier aurait en vue. Bien entendu, ce heurt contribue au succès même de toute comédie. Mais comment tout cela affecte-t-il le public? Les 
critiques et le public se réfugient souvent dans de vagues notions telles que le "sériocomique " mais ce terme appliqué à la comédie satirique m'a toujours semblé être une sorte de tentative désespérée pour tenter de défendre les revendications de vérité du satiriste alors que justement, d'autres éléments présents dans la satire indiquent clairement que nous ne devrions pas raisonner ainsi.

Un passage du Ploutos (388 av. J.-C.) d'Aristophane illustre bien cette tension entre le sérieux et le comique qui existe au sein de toute pièce satirique. Dans le débat opposant la Pauvreté à Chrémyle quant à savoir ce qui est le plus utile aux hommes - la pauvreté ou l'argent (représenté ici par Ploutos) - la Pauvreté objecte aux vers 557 et suivants :

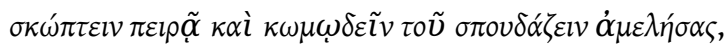

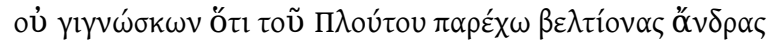

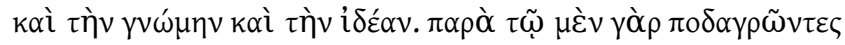

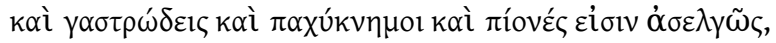

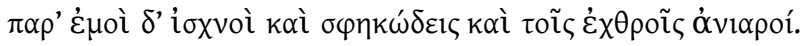

Tu essayes de railler et de me tourner en ridicule sans souci d'être sérieux ; et tu ne sais pas que, plus que Ploutos, je rends les hommes supérieurs et pour l'esprit et pour le corps. Avec lui, ils sont podagres, ventrus, épais de cuisses, gras insolemment; avec moi ils sont minces, à taille de guêpes et fâcheux pour les ennemis. (v. 557-561)

Mais Chrémyle, davantage disposé à écouter les vitupérations comiques de la Pauvreté, se refuse à toute discussion sérieuse et au vers 600 , conclut en ces termes d'une logique d'ailleurs proche de l'absurde: "Tu ne nous convaincras pas, et même si tu nous

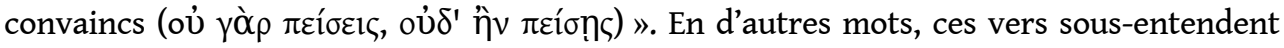
que si, au sein de la comédie satirique, une distinction peut être faite entre le " sérieux " et le « comique » et que si ce type de comédie ménage en son sein une place au sérieux, à la fin, ce sérieux ne peut jamais vraiment être pris au sérieux.

De plus, même si ce genre de scène permet au public de s'aventurer quelque peu sur ce vague territoire du "sério-comique» (le «sérieux» problème de la pauvreté par exemple, ou celui du rôle de l'argent dans la société - tous deux planant sur cette comédie qu'en font ici les personnages de cette scène), il est malgré tout impossible de connaître les réelles motivations de ce soi-disant « sérieux ». À qui s'adresse en effet ce « sérieux »? Et quel sens devons-nous lui attribuer? Est-ce un "sérieux» d'ordre didactique, informatif ou moral? Le refus de Chrémyle de se laisser convaincre par un argument logique qu'il considère cependant comme pouvant être convaincant, sous-entend que le « sério-comique » n'est au final qu'une autre prétention fallacieuse du poète satirique visant à faire rire son public, mais n'offrant en fin de compte, aucun contenu substantiel.

Cette incompatibilité fondamentale entre le sérieux et le comique au sein de la comédie satirique (et bien que le satiriste lui, affirme le contraire - et comme d'ailleurs aussi de nombreux critiques), provient donc aussi, d'après moi, de ce conflit bien particulier entre la diachronie et la synchronie qui, comme je l'ai d'ailleurs suggéré aussi, est inhérent à ce type de formes littéraires.

Nous pouvons donc également avancer que ce conflit est composé, d'une part, d'une " émotivité » synchronique quand l'auteur prétend exposer dans son œuvre une hostilité et une indignation sincères envers certains individus de son époque, et, d'autre part, d'un « intellectualisme » diachronique quand, sciemment et parfois même malicieusement, l'auteur souhaite attirer l'attention sur le cadre littéraire et historique d'après lequel il construit son monde contemporain et immédiat. 
ses «Observations finales » sur la temporalité dans le roman, Bakhtine conclut en ces termes : «En conséquence, toute pénétration dans la sphère des sens ne peut se faire qu'en passant par la porte des chronotopes $»^{22}$. Bien entendu, cela est vrai aussi pour le sens d'une œuvre satirique. Cependant, et comme nous l'avons vu dans le cas d'Aristophane, dès que nous franchissons le seuil de ce "chronotope » bien particulier, nous attend alors un monde particulièrement volatile et temporellement instable. Or c'est bien de ce tiraillement continuel entre synchronie et diachronie que naissent les différents sens de ces textes, et ce qui constitue dès lors aussi le trait distinctif d'une telle littérature.

\title{
BIBLIOGRAPHIE
}

\author{
Abréviations \\ W = West M. L., Iambi et elegi Graeci ante Alexandrum cantati. t.1 : Archilochus. Hipponax. Theognidea, \\ Oxford (2 éd.), 1989. \\ Dg = Degani E., Ipponatte : frammenti, Bologne, 2007.
}

\section{Bibliographie générale}

Baines V. et Rosen R. M. (2002), « "I am whatever you say I am” : Satiric program in Juvenal and Eminem », Classical and Modern Literature 22-2, p. 103-127.

Bakhtine M. (1978), Esthétique et théorie du roman, Paris.

Brown C. (1997), « Iambos », dans Gerber D. E. éd., A Companion to the Greek Lyric Poets, Leyde, p. 13-88.

Clay D. (2001), « The Scandal of Dionysos on Paros (The Mnesiepes Inscription E1 III) », Prometheus 27-2, p. 97-112.

Clay D. (2004), Archilochos Heros : The Cult of Poets in the Greek Polis, Washington, D. C.

Cullyer H. (2006), « Agroikia and Pleasure in Aristotle », dans Sluiter I. et Rosen R. éd., City, Countryside, and the Spatial Organization of Value in Classical Antiquity, Leyde, p. 181-217.

Degani E. (1984), Studi su Ipponatte, Bari.

Degani E. (1991), Hipponax : Testimonia et Fragmenta, Leipzig ( $2^{\mathrm{e}}$ éd.).

Degani E. (1993), « Aristofane e la tradizione dell' invettiva personale in Grecia », dans Bremer J. et Handley E. éd., Aristophane, Genève, p. 1-49.

Dover K. J. (2005), «The limits of allegory and allusion in Aristophanes », dans Cairns D. L. et Knox R. A. éd., Law, Rhetoric, and Comedy in Classical Athens, Swansea, p. 239-249.

Gerber D. éd. (1999), Greek Iambic Poetry: From the Seventh to the Fifth Centuries B.C., Cambridge, MA. 
Halliwell S. (2008), Greek laughter : a study of cultural psychology from Homer to early Christianity, Cambridge.

Hawkins T. (2008), « Lycambes gets capped », dans Katsonopoulou D., Petropoulos I. et Katsarou S. éd., Archilochos and his age, Athènes, Paros, p. 49-60.

Howes C. (1986), « Rhetorics of attack : Bakhtin and the aesthetics of satire », Genre 18, p. 215-243.

Lada-Richards I. (1999), Initiating Dionysus : Ritual and theatre in Aristophanes' Frogs, Oxford.

Möllendorff von P. (1995), Grundlagen einer Ästhetik der Alten Komödie : Untersuchungen zu Aristophanes und Michail Bachtin, Tübingen.

Rosen R. M. (1988a), Old comedy and the iambographic tradition, Oxford.

Rosen R. M. (1988b), « Hipponax, Boupalos, and the conventions of the psogos ", Transactions of the American Philological Association 118, p. 29-42.

Rosen R. M. (2007), Making Mockery : the Poetics of Ancient Satire, Oxford-New York.

Rosen R. M. (2013), « Iambos, comedy and the question of generic affiliation », dans Bakola E., Prauscella L et Telò M. éd., Greek Comedy and the Discourse of Genres, Oxford, p. 81-97.

Rotstein A. (2010), The Idea of Iambos, Oxford.

West M. (1974), Studies in Greek and elegy and iambus, Berlin-New York.

\section{NOTES}

1. Cet article est la version sensiblement remaniée de ma contribution «Aristophanes and the Pretense of Synchrony ", dans José González éd., Diachrony: Diachronic Aspects of Ancient Greek Literature and Culture, MythosEikonPoiesis, Walter de Gruyter, à paraître en 2014. Cette version en français est également traduite de l'anglais par Nathalie Ciesco.

2. Pour une définition détaillée de la «satire ", je me permets de renvoyer à Rosen 2007, p. 17-23, où je propose également une bibliographie complète. Je rappellerai donc très brièvement ici que la «satire » se rapporte à une forme littéraire bien spécifique où l'auteur se présente lui-même (ou elle-même) blessé in propria persona par certains individus ou par une certaine catégorie de personnes, ou encore par des institutions, et qui, feignant alors l'indignation moralisatrice, y répond en usant du ridicule, de la raillerie et de l'invective et en employant également tous les tropes et toutes les modalités qui s'y réfèrent.

3. Je me réfère ici d'une part à la remarque d'Alcidamas dans la Rhétorique d'Aristote (1398b11-12) observant que les gens de Paros qui en effet célébraient Archiloque, le considéraient pourtant aussi comme "blasphémateur"; et d'autre part, à l' "inscription Mnesiepes" décrivant Archiloque comme étant bien «trop iambique» (dans ses invectives, par exemple). Voir également Brown 1997, p. 46-48 ; Clay 2001, p. 97-112 ; Clay 2004, p. 16-23 ; Rosen 2007, p. 147 ; Rotstein 2010, p. 293-294.

4. Sur ce sujet, voir également Rotstein 2010, p. 326-327 ; Cullyer 2006, p. 191-196 ; Halliwell 2008, p. 317-331.

5. Sur les fragments de Lycambe, voir Brown 1997, p. 51-53 ; Hawkins 2008, p. 49-60.

6. Voir Archiloque fr. 196a W. Autres études importantes sur ce fragment d'Archiloque dans Arethusa, 9, 2, 1976.

7. Traduit de l'anglais (version anglaise : Gerber).

8. Voir Rosen 1988b, p. 35-37. 
9. Bien que l'on ne puisse guère douter de l'existence de Boupalos (voir à ce propos Degani 1984, p. 19-22), l'on ne peut en revanche affirmer avec certitude que Lycambe et ses filles ont eux, réellement existé (cf. West 1974, p. 26-27). Le doute plane d'ailleurs aussi sur l'existence des très nombreux autres personnages "secondaires" qui peuplent les œuvres de ces poètes. Par conséquent, les attaques d'un poète à l'encontre d'un personnage « fictionnel » poursuivent un objectif qui est différent de celui que visent les insultes de ce même poète envers un personnage « réel». Ces personnages fictionnels ne peuvent guère en effet, répliquer hors du contexte littéraire qui les fait vivre, c'est-à-dire alors aussi, hors de leur existence poétique.

10. Il convient de souligner ici qu'en réalité, les satiristes ont tous des prétentions littéraires mais qu'ils mettent également tout en œuvre pour nous faire croire justement, qu'ils n'en ont aucune. Au sujet de ces tensions qui surviennent entre ce prétexte d'“immédiateté " ou d'« urgence temporelle » si caractéristique de la satire, et les efforts esthétiques déployés par l'auteur satirique, je me permets de renvoyer à Baines et Rosen 2002, p. 108-113. Voir également Howes 1986, p. 216, qui pose la question autrement : « First, since satire is notoriously the genre most contaminated by the historically specific, the sub-literary, can we even think about satire as a literary genre without demanding from individual texts evidence of a sustained aesthetic transformation? Second... could satire be satire without enacting the traditional transformation of content by form to meet certain moral and aesthetic ends?".

11. Concernant ces questions, je renvoie ici à l'analyse de Juvenal, Satires, 5 et 9 que je propose dans Rosen 2007, p. 207-242.

12. Propos de table, 712A. Traduit de la version anglaise de Storey, remaniée.

13. Par exemple, les altercations entre Paphlagon et le marchand de saucisses dans Les cavaliers d'Aristophane (vers 285-302 ou vers 367-382). Voir également Rosen 2007, p. 78-91.

14. Voir ici encore Rosen 2007. Dans cette partie de mon étude, je montre en effet comment les auteurs satiriques tentent de contrôler la bienveillance de leurs différents publics.

15. Voir Rosen 1988a, p. 69-71.

16. Voir par exemple, Hipponax fr. 20, 25, $37 \mathrm{Dg}$.

17. Voir Dover 2005, p. 239-249. Bien qu'il s'intéresse davantage à l'aptitude du public à comprendre l'allégorie poétique, K. J. Dover se penche également ici sur cette éternelle question qui est de savoir en effet si les spectateurs de l'Antiquité comprenaient bien (et de quelle manière alors aussi) les stratagèmes littéraires du poète.

18. Voir Hipponax fr. 1-38 Dg. Voir Degani 1984, p. 19-25; Degani 1993, p. 1-49. Voir enfin Rosen 1988a.

19. Pour une analyse et une discussion plus détaillées, voir Rosen 2007, p. 3-42.

20. Ce célèbre passage a largement été étudié durant ces dernières décennies. Halliwell 2008, p. 211-214 offre également une bibliographie complète. Voir aussi Lada-Richards 1999, p. 223-225. Enfin, je renvoie également à Rosen (à paraître).

21. Bakhtine 1978, p. 395, «Formes du temps et du chronotope dans le roman ». La position de Bakhtine envers la satire demeure quelque peu complexe, voire équivoque, dans la mesure où luimême semble incommodé par les tendances «monologiques" du discours polémique. De plus, Bakhtine n'envisage la satire que comme une modalité rudimentaire, grossière et primitive qui ne peut porter ses fruits que lorsqu'elle est incorporée au roman (bien qu'il s'intéresse davantage à la diversité carnavalesque et à la polyphonie de ce qu'il nomme la satire « Menippean »). À ce sujet, voir Howes 1986, p. 241 : «For Bakhtin, satire becomes the novel when it leaves off its polemical concern with the contemporary, and strives instead to harmonize its many voices in an aesthetically rounded chorus ". Concernant l'application du chronotope bakhtinien à l'analyse de la comédie d'Aristophane, voir Möllendorff 1995.

22. Bakhtine 1978, p. 398, « Observations finales». 


\section{RÉSUMÉS}

Les genres littéraires de la satire se trouvent continuellement confrontés à la question de l'efficacité : cette littérature est fortement ancrée dans ses lieux et temps propres : son succès repose sur l'effet qu'elle peut avoir sur les cibles ainsi ridiculisées et invectivées. Bref, ces genres sont censés faire quelque chose. Mais, en fait, on ne peut les lire sous cet angle qu'à l'époque à laquelle ces œuvres ont été composées. Or quelle sorte d'efficacité peut exister pour cette littérature dans les époques ultérieures, quand les cibles de l'invective ne sont plus guère connues et n'ont certainement aucune importance réelle pour les lecteurs? Cet essai examine quelques œuvres de l'iambe grec et de la comédie ancienne pour soutenir que, tout d'abord, la satire littéraire, plus que tous les autres genres et modes, dépend d'une tension continuelle entre deux perspectives - celle de la synchronie (c'est-à-dire, l'œuvre dans son époque propre) et celle de la diachronie (l'œuvre vue par la postérité) - et qu'ensuite, il faut bien comprendre cette tension pour comprendre la « signification » de la satire.

Satirical literary genres are continually faced with the question of efficacy: such literature is highly localized in place and time, and thrives on the pretense that their invective and ridicule will have an effect on the human targets and events they attack. These genres are supposed, in short, to do something. But such a pretense can really only be entertained at the time when the works were actually produced. For what kind of efficacy can exist for such literature in subsequent chronological eras when the targets of invective are barely known and certainly have no real significance for the lives of their readers? This paper discusses various works of the Greek iambus and old Comedy in support of the argument first, that literary satire, more than any other genre or mode, relies on a continual tension between two perspectives-the synchronic (the work situated in its own time) and the diachronic (the work considered in posterity)-and next that understanding this tension is critical to an understanding of 'meaning' in satirical literature.

\section{INDEX}

Keywords : satire, Greek iambus, diachronic, synchronic, Archilochus, Hipponax, Aristophanes

Mots-clés : satire, poésie iambique, diachronie, synchronie, Archiloque, Hipponax, Aristophane

\section{AUTEUR}

RALPH M. ROSEN

University of Pennsylvania 